\title{
MODEL-DRIVEN STATISTICAL ANALYSIS OF HUMAN GAIT MOTION
}

\author{
Jang-Hee Yoo, Mark S. Nixon and Chris. J. Harris \\ Department of Electronics and Computer Science \\ University of Southampton, Southampton SO17 1BJ, UK \\ [jhy00r|msn|cjh]@ecs.soton.ac.uk
}

\begin{abstract}
We describe a new method for analyzing and extracting human gait motion by combining statistical methods with image processing. The periodic motion of human gait is modeled by trigonometric-polynomial interpolant functions. The gait description is derived by topological analysis guided by medical studies that select areas from which joint angles are derived by regression analysis. Then, the interpolant functions are fitted to the gait data and whilst showing fidelity to earlier medical studies, also show recognition capability. As such, a new combination of medical knowledge, image processing and regression analysis can be used to label human motion in image sequences.
\end{abstract}

\section{INTRODUCTION}

Recent interest in computer vision has emerged which deals with the analysis of image sequences involving humans. This interest is motivated by the various application domains [1]: visual surveillance, virtual reality, athletic performance analysis, video database browsing, modelbased coding, and biometrics. Moreover, human motion analysis has many challenging issues, because the highly flexible structure and self-occlusion of the human body mandates complicated processes for motion segmentation and analysis. The motion of the human body is known as a form of non-rigid and articulated motion [2]. Human gait motion is one of the most universal and complex of all human activities [3], and it can be decomposed into periodic components within a gait cycle.

In computer vision, recognition by the gait has recently been investigated [4]. The human gait is a pattern of human locomotion and can be described by kinetic or kinematic characteristics [2,6]. Also, gait is often modeled as harmonic motion such as a pendulum $[4,5]$. In fact, gait has many degrees of freedom, and is thus a more complex and chaotic motion. The gait measurement systems in most medical examination have used markers that represent the observed movement in the form of 3D trajectories that translate into kinematic variables such as body movements and joint angles [7]. However, using markers needs intrusive and expensive specialized hardware and requires contact with a subject and concerns overt rather than covert use. To enable greater application capability, markerless systems are required.

Here we propose a new method for modeling, analyzing and extracting human gait by combining statistical methods with image processing. Gait is interpolated using trigonometric polynomials and analyzed by using phasespace portrait and angle-angle cyclogram for periodicity detection. To extract gait angles, border data of a body segmented by using anatomical knowledge are extracted from the body contour, that is preprocessed by image processing techniques. The gait angles and their associated with certainty factor are then approximated by linear regression model. Also, trajectory-based kinematic features are calculated from image sequences by analyzing the gait motion. Results show that the new approach works successfully, in comparison with medical data acquired by a marker-based system, and should therefore prove suited to the suggested application domains.

\section{HUMAN GAIT AS PERIODIC MOTION}

A period of the human gait cycle exists between the successive heel-strikes. The motion period and gait frequency are the time taken by a step and the number of steps taken per second, respectively. Murray [8] considers human gait as "a total walking cycle" - the action of walking can be thought of as a periodic signal. Basically, each gait cycle is separated into two distinct periods: the stance and the swing phase. The stance phase is the entire period during which the foot is on the ground, and the swing phase begins as the foot is lifted from floor (toe-off). The period of floor contact $60 \%$ for stance and $40 \%$ for swing [9].

However, in kinematic analysis human gait is usually characterized by the joint angles between body segments and their relationships to the events of the gait cycle [6]. Statistical analyses of the gait relationships typically use continuous curves of the time series data measured over the gait cycle. Medical research [8] has shown that the pattern of gait motion is approximately sinusoidal in nature. Trigonometric functions with period $2 \pi$ are naturally suited to estimating a gait curve by time series data. Consequently, an assumed functional relationship between periodic gait motion and time can be modeled by interpo- 
lation of trigonometric polynomials. Also, further gait motion can be predicted by the periodicity of this interpolation model. An $n^{\text {th }}$-order trigonometric polynomial interpolant function is [10]

$$
\begin{aligned}
y_{n}(x)= & a_{0}+a_{n} \cos (2 \pi n x) \\
& +\sum_{k=1}^{n-1}\left[a_{k} \cos (2 \pi k x)+b_{k} \sin (2 \pi k x)\right]
\end{aligned}
$$

where the $a_{0}, a_{n}, a_{k}$ and $b_{k}$ are unknown curve-specific coefficients. As $n \rightarrow \infty, y_{n}(x)$ tends to the Fourier series.

The interpolation much (equally-spaced) data by trigonometric polynomials can make for very accurate results. Figs.1(a) and (b) show the curves for joint angles of the thigh and knee motion during one gait cycle. Here, the points are the medical data from anatomical markers in a limb segment [11], and the lines are the curves that result from using $4^{\text {th }}$-order trigonometric-polynomial interpolants.

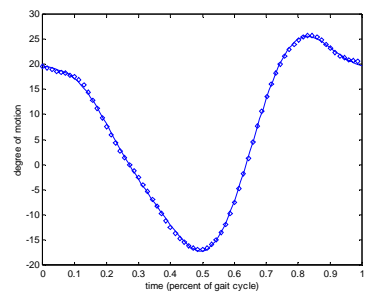

(a)Thigh Angles vs. Time

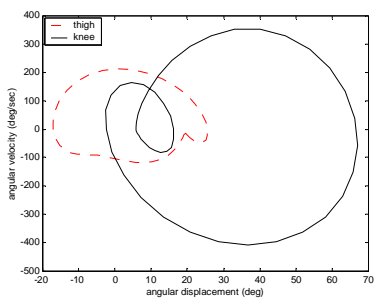

(c) Phase-Space Portrait

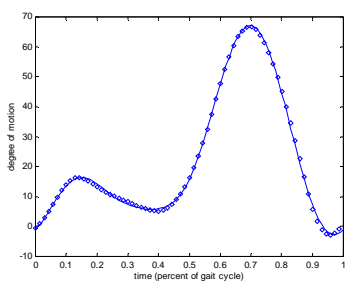

(b) Knee Angles vs. Time

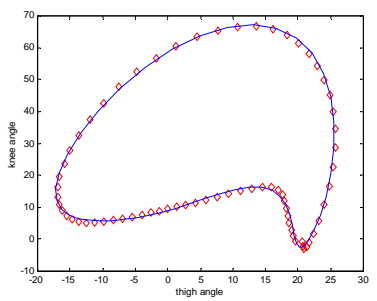

(d) Thigh-Knee Cyclogram FIGURE-1: Gait Angles during One Gait Cycle
The phase-space portrait is a useful method to represent the periodic motion analysis. Figs. 1(c) and (d) are the phase-space portraits of angular velocity versus displacement and angle-angle cyclogram for the thigh and knee motion. Using angle vs. angle plots rather than angle vs. time curves improves understanding of gait HOW???.

\section{EXTRACTING HUMAN GAIT MOTION}

\subsection{Extracting the Body Contour}

To detect the human body, background subtraction [12] using an edge difference image with noise filtering is employed to handle any change in illumination. The histogram projection profiles are analyzed to estimate the position of a human body in the edge difference image. Figs. 2(a) and (b) show a real scene image with $384 \times 288$ gray- scale and detected human body region by prior knowledge such as size and shape.

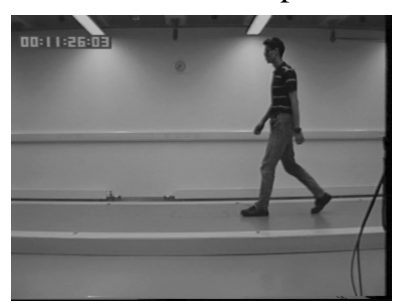

(a) Grey-Scale Image

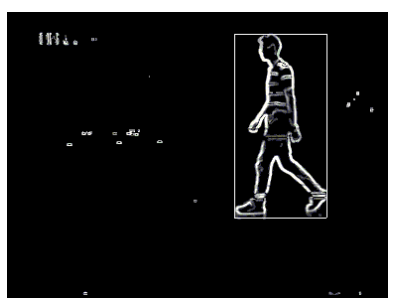

(a) Body Detection
FIGURE-2: Detecting the Human Body

Thresholding and morphology is then used to extract the contour of a detected human body. A thresholding method based on similarity measures between the background and the current image is used. Let $I_{r b}(x, y)$ and $I_{r c}(x, y)$ be the brightness of a pixel with coordinate $(x, y)$ in raw background image $\left(I_{r b}\right)$ and raw current image $\left(I_{r c}\right)$. Then, the similarity $\Theta(x, y)$ at coordinate $(x, y)$ is calculated by

$$
\Theta(x, y)=\left|I_{r b}-I_{r c}\right|
$$

Similarity values close to zero imply a high probability of being background; conversely, large values of similarity imply high probability as an object. Therefore, the binary image $I_{b}(x, y)$ is thresholded as

$$
I_{b}(x, y)=\left\{\begin{array}{cc}
1 & \text { if }(\Theta(x, y)>\tau) \wedge\left(I_{r c}(x, y)>\eta\right) \\
0 & \text { otherwise }
\end{array}\right.
$$

where $\tau$ is a tolerance value, and $\eta$ denotes a global threshold value. Finally, the body contour is determined by the arithmetic subtraction followed by dilation and erosion. Thresholding is a simple image segmentation method, and erosion will remove the outer layer of pixels from an object. However, this method is very effective and useful for small and low-resolution images.

\subsection{Extracting the Gait Angles}

We consider thigh (or hip) and knee angles for analyzing human gait motion. These angles are extracted from border data of the body contour by linear regression. To extract the border data, the expected horizontal knee position $(0.285 H$ for a body height $H)$ is determined by using the known body properties $[11,13]$. However, the knees have the largest movement of all parts of the leg. Therefore, five horizontal lines around the expected knee position are scanned. Also, the vertical position of the pelvis and ankles was set by study of anatomical data $[11,13]$ to be $0.480 H$ and $0.039 H$, respectively. Consequently, the border data for the thigh are collected by pixel positions between the knees and the pelvis at the front and rear boundaries. The boundary data for shin are collected from positions between knees and ankles. Fig. 3(a) shows the expected joint positions and extracted border pixels from 
the body contour determined by our new approach. Fig. 3(b) shows the extracted border data with lines selected by least-squares analysis superimposed. Data near joint positions is masked from these calculations.

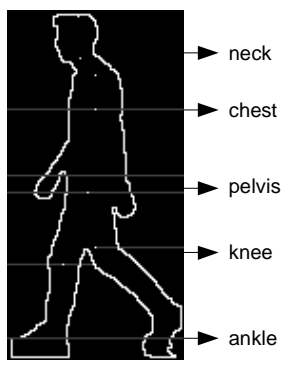

(a) Body Contour

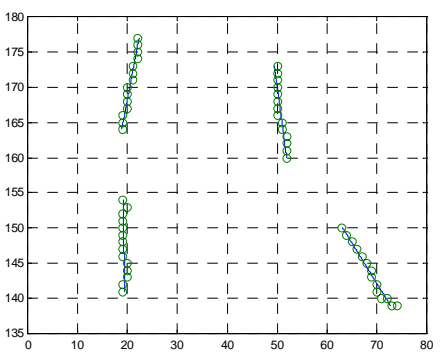

(b) Border Data and Line Fitting

\section{FIGURE-3: Extracting Gait Angles by Least Squares}

Now we can calculate the thigh (or hip) and leg angles by linear regression using the border data. The angles $\theta_{j, k}$ of joint $j$ at image frame $k$ can be calculated by the slope of the lines in linear regression equation as [14]

$$
\theta_{j, k}=\sum_{i=1}^{n}\left(y_{i}-\bar{y}\right)\left(x_{i}-\bar{x}\right) / \sum_{i=1}^{n}\left(x_{i}-\bar{x}\right)^{2} .
$$

Also, certainty factor (CF) $\gamma_{j, k}$ of the angle $\theta_{j, k}$ can be determined by the correlation coefficient as [14]

$$
\gamma_{j, k}=\sum_{i=1}^{n}\left(y_{i}-\bar{y}\right)\left(x_{i}-\bar{x}\right) / \sqrt{\sum_{i=1}^{n}\left(y_{i}-\bar{y}\right)^{2} \sum_{i=1}^{n}\left(x_{i}-\bar{x}\right)^{2}}
$$

where $n$ is the number of the points. In general, a segmented image is highly susceptible to noise in the object boundary, and the border data can include spurious pixels. Therefore, a weighted moving average is employed to reduce the influence angles measured in noisy frames as

$$
\theta_{j}(n)=\sum_{k=n-q}^{n+q}\left(\gamma_{j, k} \bullet \theta_{j, k}\right) / \sum_{k=n-q}^{n+q} \gamma_{j, k}
$$

where $q$ is a size of moving window, here set to 2. By definition, the joint angles are relative, thus the relative angles in each joint were approximated from the measurement values of the angles with the certainty factor. Figs. 4(a) and (b) show the definition of the measurement angles and relative joint angles. In normal walking, the trunk can be considered to be almost vertical, and the knee angles can be approximated by the thigh and leg angles. The CF for knee angle is updated by MYCIN's CF update rule [15] as

$$
\gamma_{\text {knee }, k}=\left(\gamma_{\text {thigh }, k}+\gamma_{\text {leg }, k}\right)-\left(\gamma_{\text {thigh }, k} \times \gamma_{\text {leg }, k}\right) .
$$

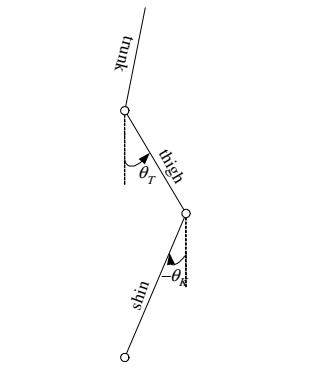

(a) Extracted Angles

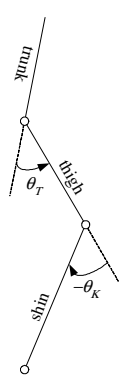

(b) Relative Angles
FIGURE-4: Definition of Joint Angles

\section{RESULTS}

Human gait motion can be described in a compact form, as a sequence of the joint parameters. In the experiments, three different humans with around 30 consecutive images for each gait cycle were analyzed. A set of the gait data extracted from image sequences provides potentially valuable time-dependent patterns as a gait time series. The gait angles are approximated by Eqns. (4), (5), (6) and (7), and the lines are interpolation curves from Eqn. (1). Fig. 5 shows the results of measuring human gait angles and periodicity analysis during a gait cycle. These are consistent with medical data of Figs. 1(a) and 1(b) and differ between subjects. The human gait is a kind of periodic motion, especially when walking laterally. In the figures (c) and (d), we can find a periodicity of the human gait motion. We can also predict a gait motion by using the

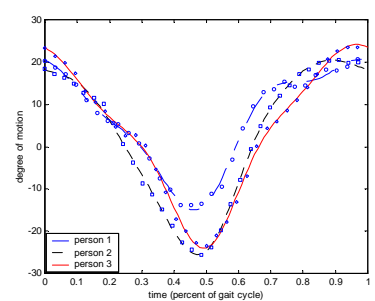

(a) Thigh Angles

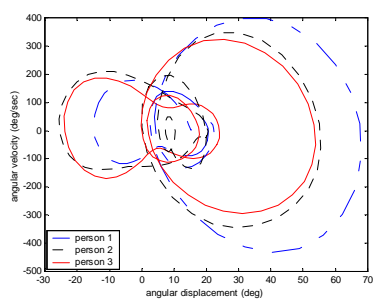

(c) Phase-Space Portrait

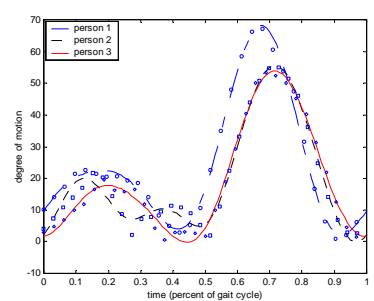

(b) Knee Angles

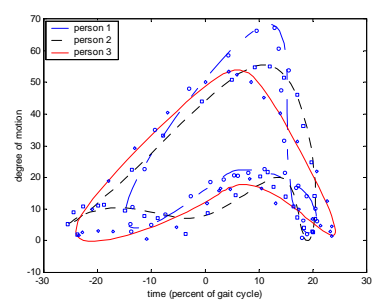

(d) Thigh-Knee Cyclogram
FIGURE-5: Periodicity Analysis for Gait Angles

phase-space portrait and angle-angle cyclogram. As experienced in medical studies, the thigh angles progressively extend after mid stance, and the initial and final degrees have almost the same values during the gait cycle. 
To compare consistency of the gait motion, we use two different image sequences for each person. Fig. 6 shows the experimental results for comparison analysis of the gait motion. In Fig. 6(a), the small curves on the left region show the thigh motion, whilst the large curves on the right show the knee motion. Clearly, the knee motion is characterized by a large range of motion. Also, person 2 and person 3 have very little difference between the curves from two different image sequences. The two curves of person 1 do not match as well as those of the other subjects, because person 1 due to9 their particular style of clothing.

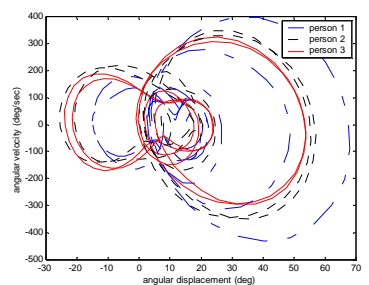

(a) Phase-Space Portrait

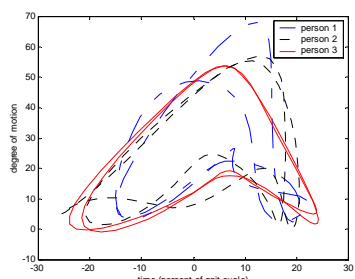

(b) Thigh-Knee Cyclogram
Figure-6: Comparing Different Subjects

To identify human by a gait motion, an enhanced backpropagation algorithm is employed as a classifier, and the coefficients for trigonometric polynomial interpolation and the trajectory-based features for gait kinematics are used as input data. We have mainly used kinematic features such as average linear and angular velocities selected based on heuristic knowledge. The features are extracted from the image sequence during a time periodic. Though the recognition rate here was $100 \%$ we have used a very small set of data for classification, thus making meaningful comparisons of recognition performance impossible. Naturally we seek to extend the analysis in future.

\section{CONCLUSIONS}

We have described a new method for modeling, analyzing and extracting the gait motion by statistical methods and computer vision. The gait motion can be characterized as having a periodic component. The periodicity of human gait motion was modeled by interpolation of trigonometric polynomials. The phase-space portrait and angle-angle cyclogram are used for periodicity analysis and prediction model of the gait motion. Also, the gait angles with certainty factor are extracted by linear regression model from the human body contour in known body topology. Trajectory-based kinematic features such as linear and angular velocities are extracted from the image sequences. Then, an enhanced back-propagation algorithm is employed to classify the gait features. As such, this new approach appears to recognize the body's structure and its motion. In future we seek to clarify the technique in terms of applica- tion capability as well as analyzing a larger database of subjects.

\section{REFERENCES}

[1] D. M. Gavrila, "The Visual Analysis of Human Movement: A Survey", Computer Vision and Image Understanding, 73(1), pp.82-98, Jan. 1999.

[2] J. K. Aggarwal, Q. Cai, W. Liao, and B. Sabata, "Nonrigid Motion Analysis: Articulated and Elastic Motion", Computer Vision and Image Understanding, 70(2), pp.142-156, May 1998.

[3] H. Sadeghi, P. Allard, F. Prince, and H. Labelle, "Symmetry and Limb Dominance in Able-Bodied Gait: A Review", Gait and Posture, 12(1), pp.34-45, 2000.

[4] M. S. Nixon, J. N. Carter, D. Cunado, P. S. Huang, and S. V. Stevenage, "Automatic Gait Recognition", in BIOMETRICS - Personal Identification in Networked Society, A. K. Jain, et al. Eds., pp.231-249, Kluwer Academic Publishers, 1999.

[5] R. Cutler and L. Davis, "Robust Real-Time Periodic Motion Detection, Analysis, and Applications", IEEE Transaction on Pattern Analysis and Machine Intelligence, pp.781796, 22(8), August 2000.

[6] S. J. Olney and C. L. Richards, "Hemiparetic Gait Following Stroke. Part I: Characteristics", Gait and Posture, 4(2), pp.136-148, 1996.

[7] R. F. M. Kleissen, J. H. Buurke, J. Harlaar, and G. Zilvold, "Electromyography in the Biomechanical Analysis of Human Movement and its Clinical Application", Gait and Posture, 8(2), pp.143-158, 1998.

[8] M. P. Murray, A. B. Drought, and R. C. Kory, "Walking Pattern of Normal Men", Journal of Bone and Joint Surgery, 46(2), pp.335-360, 1964.

[9] J. Perry, Gait Analysis: Normal and Pathological Function, Slack, 1992.

[10] R. L. Burden and J. D. Faires, Numerical Analysis, $7^{\text {th }}$ (eds.), Pacific Grove, 2001.

[11] D. A Winter, The Biomechanics and Motor Control of Human Movement, $2^{\text {nd }}$ (Eds.), John Wiley \& Sons, 1990.

[12] T. Horprasert, D. Harwood, and L. S. Davis, "A Statistical Approach for Real-Time Robust Background Subtraction and Shadow Detection", Proc. IEEE Frame-Rate Workshop, Kerkyra, Greece, Sept. 1999.

[13] W. T. Dempster and G. R. L. Gaughran, "Properties of Body Segments Based on Size and Weight", American Journal of Anatomy, 120, pp.33-54, 1967.

[14] G. R. Terrell, Mathematical Statistics: A Unified Introduction, Springer, 1999.

[15] B. G. Buchanan and E. H. Shortliffe, Rule-Based Expert Systems: The MYCIN Experiments of the Stanford Heuristic Programming Project, Addison-Wesley, 1984. 